\title{
Accuracy of a Rapid Diagnostic Test for the Presence of Direct Oral Factor Xa or Thrombin Inhibitors in Urine-A Multicenter Trial
}

\author{
Job Harenberg ${ }^{1,2}$ Jan Beyer-Westendorf ${ }^{3,4}$ Mark Crowther $^{5} \quad$ Jonathan Douxfils $^{6,7}$ Ismail Elalamy ${ }^{8,9}$ \\ Peter Verhamme $^{10}$ Rupert Bauersachs ${ }^{11}$ Svetlana Hetjens ${ }^{12}$ Christel Weiss $^{12}$
}

${ }^{1}$ DOASENSE GmbH, Heidelberg, Germany

2 Ruprecht Karls University of Heidelberg, Heidelberg, Germany

${ }^{3}$ Division Hematology, Thrombosis Research Unit, Department of Medicine I, University Hospital “Carl Gustav Carus” Dresden, Dresden, Germany

${ }^{4}$ Department of Haematology, King's College London, London, United Kingdom

${ }^{5}$ Thrombosis and Atherosclerosis Research Institute, Department of Medicine, McMaster University, Hamilton, Ontario, Canada

${ }^{6}$ Department of Pharmacy, Namur Thrombosis and Hemostasis Centre, Namur Research Institute for Life Sciences, University of Namur, Namur, Belgium

${ }^{7}$ QUALIblood, Namur, Belgium

8 Service d'Hématologie Biologique Hôpital Tenon, Hôpitaux Universitaires Est Parisien, Assistance Publique Hôpitaux de Paris, Paris, France

${ }^{9}$ Department of Obstetrics and Gynecology, The First I.M. Sechenov Moscow State Medical University, Moscow, Russia

10 Department of Cardiovascular Sciences, Vascular Medicine and Haemostasis, University of Leuven, Leuven, Belgium

${ }^{11}$ Department of Vascular Medicine, Klinikum Darmstadt GmbH, Darmstadt, Germany

12 Department of Statistics, Medical Faculty Mannheim, Ruprecht Karls University of Heidelberg, Mannheim, Germany
Address for correspondence Job Harenberg, MD, DOASENSE GmbH, Waldhofer Strasse 102, D-69123 Heidelberg, Germany (e-mail: j.harenberg@doasense.de).

Thromb Haemost 2020;120:132-140.
Abstract
Keywords
- direct oral anticoagulants
- point-of-care test
- apixaban
- edoxaban
- rivaroxaban
- dabigatran

The rapid determination of the presence of direct oral anticoagulants (DOACs) in a patient remains a major challenge in emergency medicine and for rapid medical treatment decisions. All DOACs are excreted into urine. A sensitive and specific point-ofcare test has been developed to determine whether they are present in patient urine samples. This prospective multicenter study aimed to demonstrate at least $95 \%$ correct positive and negative predictive results for factor $X a$ and thrombin inhibitors in urine samples using DOAC Dipstick pads compared with liquid chromatography-tandem mass spectrometry (LC-MS/MS) (NCT03182829). Nine hundred and fourteen subjects were included and 880 were evaluated per protocol (factor Xa inhibitors apixaban, edoxaban, and rivaroxaban: $n=451$, thrombin inhibitor dabigatran: $n=429$ ) at 18 centers. The sensitivity, specificity, accuracy, and predictive values and agreement between methods for determination of factor $X a$ inhibitors were at least noninferior to $95 \%$ with a $0.5 \%$ margin and of thrombin inhibitor superior to $97.5 \%$. These results were compared with LC-MS/MS results in the intention-to-analyze cohort (all $p<0.05$ ). The receiver operating curve showed $c$-values of 0.989 (factor Xa inhibitors) and 0.995 received

August 14, 2019 accepted after revision September 7, 2019
DOI https://doi.org/ $10.1055 / \mathrm{s}-0039-1700545$. ISSN 0340-6245. (c) 2020 Georg Thieme Verlag KG Stuttgart · New York
License terms

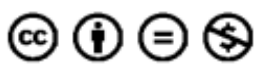


(thrombin inhibitor). Visual evaluation of the factor Xa and thrombin inhibitor pads was not different between centers. Qualitative determination of both types of DOACs was accurate using the DOAC Dipstick compared with using LC-MS/MS. The high predictive values may impact laboratory and clinical decision-making processes.

\section{Introduction}

Direct oral anticoagulants (DOACs) have become the preferred treatment for nonvalvular atrial fibrillation and venous thromboembolism. ${ }^{1,2}$ In emergency situations, DOACs may need to be detected quickly, but this is not always feasible; thus, confirmation of the presence or absence of DOACs remains a major challenge. ${ }^{3-5}$ Various tests are in different stages of development. ${ }^{6-8}$ Emergency situations with a need for point-of-care testing for the presence or absence of DOACs include emergent or urgent major surgical interventions, clinically relevant bleeding, or thrombotic episodes with known or unknown anticoagulant therapy, as well as the evaluation of unconscious patients or those unable to inform clinicians about their anticoagulant therapy. ${ }^{9,10}$ Knowledge of the presence of a DOAC may inform use of expensive reversal agents, which would not be indicated in a patient without detectable DOAC exposure.

The in vitro diagnostic DOAC Dipstick was developed based on the rationale that DOACs are excreted into urine. ${ }^{11}$ Factor Xa inhibitors and thrombin inhibitors are detected on different DOAC Dipstick pads, each containing immobilized reagents that specifically interact with the respective DOAC. Matrix plasma proteins and cells are not present in urine samples, unlike in blood or plasma, so cannot interact with DOACs during the test and interfere with the DOAC Dipstick results. ${ }^{12}$

A multicenter study was conducted to assess the accuracy of the test in detecting direct oral factor Xa inhibitors (DXI) and direct oral thrombin inhibitor dabigatran (DTI) in urine samples taken from DOAC-treated patients. Testing was performed in an actual point-of-care setting and color identification results were compared with liquid chromatography-tandem mass spectrometry (LC-MS/MS) from urine samples.

\section{Subjects and Methods}

\section{Design of the Study}

In this prospective, open-label, controlled, nonrandomized, multicenter study performed in Germany, subjects treated with a DXI (apixaban, edoxaban, and rivaroxaban) or the DTI dabigatran were included (NCT03182829). The DOAC Dipstick contains two separate pads for testing DXI and DTI. The reagents that are immobilized on the pads are specific for DXI or DTI and do not interact with each other. Therefore, the thrombin pad serves as a negative control for the factor Xa inhibitor test and vice versa. ${ }^{7}$ We did not include a group of subjects not taking any DOAC.

Inclusion criteria were: (1) full understanding of the study and ability to follow the instructions, (2) signed and dated written informed consent, (3) age $\geq 18$ years, and (4) therapy with either apixaban, edoxaban, rivaroxaban, or dabigatran for at least 1 week. Exclusion criteria were inability to provide urine samples, inability to understand the informed consent, and severe mental disability. The study protocol was approved by the primary ethics committee and the local ethics committees of the participating centers (see section "Participating Centers" below) according to the Declaration of Helsinki 1964. Subjects were included in the study after they signed the informed consent form.

The subjects were recruited from 18 outpatient care units specialized in oral anticoagulation. The date and time of their last DOAC intake before urine collection was recorded and subjects were asked to provide a urine sample in a propylene container $(100 \mathrm{~mL}$ container with integrated transfer unit for closed urine transfer in the V-urine vacuum system, with lid assembled and safety label; Sarstedt AG, Nuermbrecht, Germany). Demographic data (age, sex, and body weight), DOAC type, dose and start date of DOAC therapy, indication for anticoagulation, additional diseases relevant to DOAC therapy, and additional medication relevant to DOAC therapy were documented by the study personnel in the case report form. The barcode of patient's urine sample was attached to the case report form for patient identification.

\section{Methods}

\section{Preparation of Urine Samples}

Urine samples $(4 \mathrm{~mL})$ were transferred into polyethylene terephthalate tubes (V-Monovette Urine Z 4mL, Sarstedt AG) using plastic syringes. The DOAC Dipstick test was performed on the urine sample at the time of collection by site personnel as described below. Next, barcodes were attached to the duplicate samples and recorded in the case report form. The samples were frozen immediately at $-25^{\circ} \mathrm{C}$, transferred without interrupting the cold chain to the central laboratory (MVZ Limbach and colleagues, Heidelberg, Germany) for LC-MS/MS analysis.

\section{Performance of DOAC Dipstick Test}

The test procedure has been described in detail previously. ${ }^{7}$ In short, the test strip is dipped into urine or control samples for 2 to 3 seconds until all test pads are covered by urine. Then, the test strip is placed on a flat surface at room temperature with the pads facing upwards. Pads are left for 10 minutes without contact with the table or other surfaces. The results are then compared visually with the reference scale (-Supplementary Fig. S1, available in the online version) attached to the test strip container.

Site observers at each center had to pass a standardized training program in the identification of test pad colors during the initiation visit. The visualized colors were documented on the scheme in the case report form. The rating scale contained 
the following possibilities: negative, positive + , and positive ++ for the factor Xa and thrombin inhibitor pads, normal and abnormal for the urine color pad, and low, normal + , and normal ++ for the creatinine pad (-Supplementary Fig. S1, available in the online version). The cutoff of pads of creatinine was $0.25 \mathrm{~g} / \mathrm{L}$ for "low" versus "normal" that corresponds to about $30 \mathrm{~mL} / \mathrm{min}$ creatinine clearance. Site personnel with known red-green blindness cannot perform the test. After interpreting the result, the test strips were discarded.

\section{Liquid Chromatography-Mass Spectrometry of DOACs in Urine}

LC-MS/MS was performed as previously described. ${ }^{7,13}$ The lower limit for quantifying DOACs was $<4 \mathrm{ng} / \mathrm{mL}$. The DOAC concentration in urine of subjects with chronic use of DOACs is 10- to 50-fold higher than in plasma. ${ }^{11-13}$ The threshold of $30 \mathrm{ng} / \mathrm{mL}$ in urine for positivity assumes that, in subjects with chronic use of DOAC, urine levels of the drug of interest are always above the levels in plasma. The $30 \mathrm{ng} / \mathrm{mL}$ threshold in plasma has been proposed for safe hemostasis by several experts. ${ }^{14-16}$ Using the same threshold for a positive result in urine samples was therefore a very conservative approach in the present study.

Further, if LC-MS/MS determined the level of one of the four DOACs to be $\geq 30 \mathrm{ng} / \mathrm{mL}$, a positive visual evaluation of the appropriate pad was counted as correct positive and a negative visual evaluation was counted as false negative, respectively (-Fig. 1). If LC-MS/MS determined the DOAC to be present at a concentration of $<30 \mathrm{ng} / \mathrm{mL}$, both the Xa pad and the thrombin inhibitor pad should have been interpreted as negative; thus, a negative visual evaluation was counted as correct negative and a positive visual evaluation as false positive, respectively (-Fig. $\mathbf{1}$ ).

\section{Endpoints}

The primary endpoint of the study was to analyze the true positive and true negative rate of the factor Xa inhibitor and thrombin inhibitor DOAC Dipstick test compared with the results obtained by LC-MS/MS.

Intercenter variability was analyzed because visual identification of the test pad colors could not be quantitatively analyzed.

\section{Documentation of the Data}

Biographic data, indication for DOAC therapy, DOAC taken, inclusion and exclusion criteria, concomitant diagnoses and medications, visual evaluation of the DOAC Dipstick test, and urine transfer to specific tubes for LS-MS/MS analysis were documented in a specific case report form. Two documentation assistants, following the four-eye principle, entered data into Excel 2017. Data were then transferred to SAS release 9.4 (see below) for statistical analysis.

\section{Sample Size Determination}

The study hypothesis was that the proportions of correct positive and correct negative test results using the DOAC Dipstick would be at least $95 \%$ assuming an equivalence limit of $2.5 \%$ conducting a one-sided test with a power of 0.80 and a significance level of $p<0.05$. The sample size calculation for a binomial test used the ONESAMPLEFREQ statement in the SAS procedure SAS POWER. ${ }^{17}$ This required 384 evaluable subjects per DXI and DTI group. To account for a predicted dropout rate of $12 \%$, we increased the sample size to $n=440$ subjects per test group (SAS procedure PROC POWER, SAS Institute Inc., Cary, North Carolina, United States, release 9.4). If the level of significance was obtained for sensitivity, specificity, accuracy, negative or positive predictive value, and agreement between methods (kappa value), then testing was conducted using a proportion of $97.5 \%$. If any parameter was not significant, a noninferiority test was added including a margin of equivalence of $0.5 \%$ that was regarded as clinically acceptable.

Quantitative variables were presented as mean values and standard deviations or as mean values and 95\% confidence intervals. The DOAC concentrations were presented as medians and quartiles. Qualitative data were presented as absolute and relative frequencies. "Positive + " and "positive ++ " results for factor Xa and thrombin inhibitors were combined into "positive" for the analysis.

McNemar tests were conducted to compare the sensitivity, specificity, accuracy, negative predictive value, positive predictive value, and the probability of detecting both medications. The kappa coefficients were calculated to determine the strength of agreement between DOAC Dipstick and LCMS/MS methods. The intercenter variability was assessed with the chi-square test. Results were considered significant at a $p$-value of $<0.05$.

\section{Results}

During the study period (August 2018-April 2019), 914 subjects were included, of which 880 subjects were evaluable for the analysis (451 in the DXI group and 429 in the DTI group) ( - Table $\mathbf{1}$ ). The numbers and reasons for dropout are given in -Fig. 1 .

\section{Demographic Data}

Demographic data, the number of subjects treated with apixaban, edoxaban, rivaroxaban (DXI group), and dabigatran (DTI group), comorbidities, and comedications are listed in -Table 1.

\section{Results of LC-MS/MS}

The urine concentrations of apixaban, edoxaban, rivaroxaban, and dabigatran are given in - Table 2 . Levels determined by LC-MS/MS are higher in urine compared with those expected in plasma.

\section{Primary Endpoint}

\section{Per-Protocol Evaluation}

Factor Xa inhibitor DOAC Dipstick test: In the DXI group, the factor Xa inhibitor pad was evaluated in 435/452 cases (96.2\%) as correct positive and in $17 / 452$ cases (3.8\%) as false negative after comparison with the LC-MS/MS results. In the DTI group, the factor Xa inhibitor pad was evaluated in 421/ 


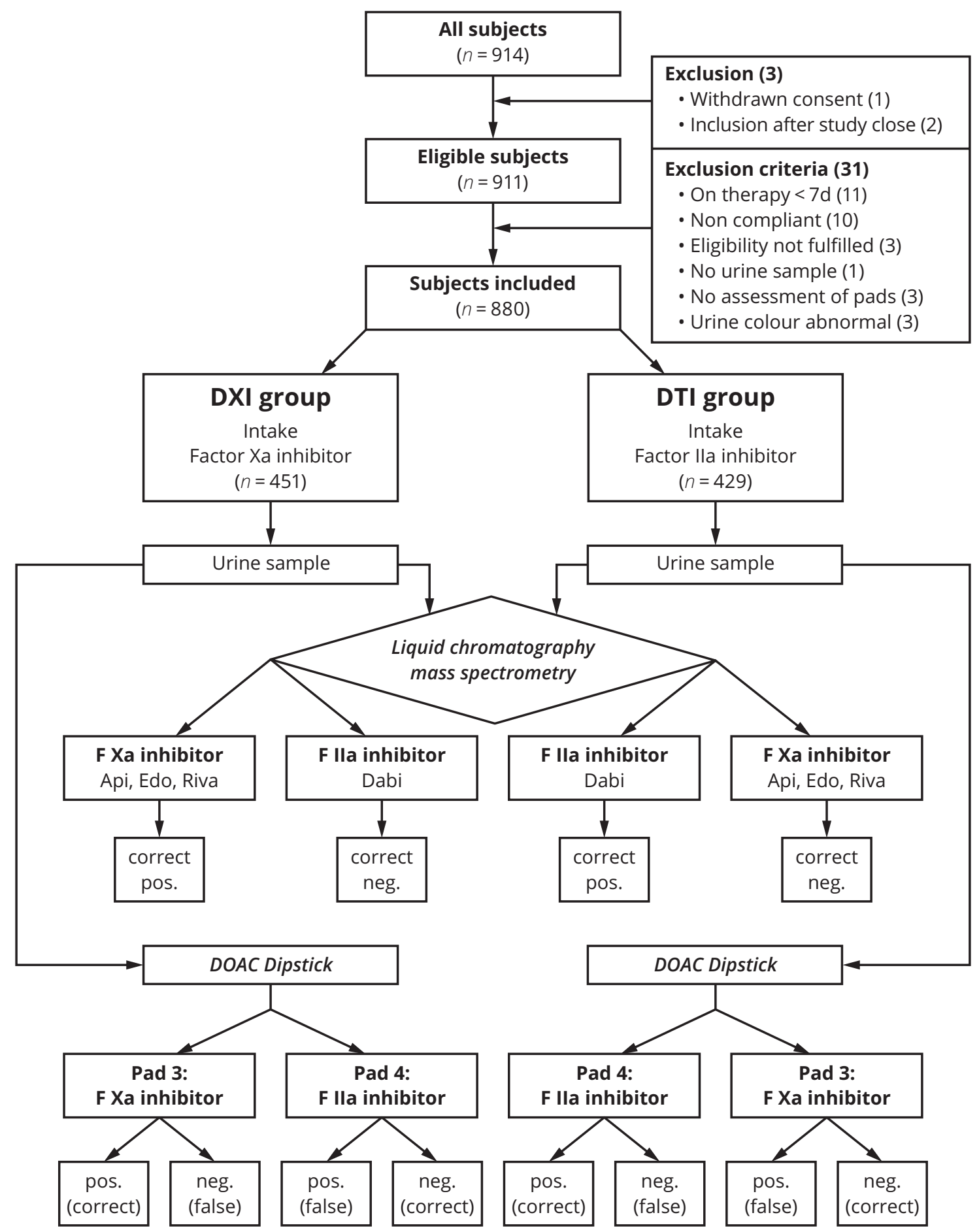

Fig. 1 Study flowchart showing the subjects included in the study and direct oral anticoagulant (DOAC) determination in urine samples using the DOAC Dipstick test and liquid chromatography-tandem mass spectrometry (LC-MS/MS) to detect correct and false visual evaluation of factor Xa inhibitor pads and thrombin inhibitor pads after incubation in urine samples taken from subjects in the oral direct factor Xa inhibitor (DXI) group and the oral thrombin inhibitor (DTI) group. Api, apixaban; Edo, edoxaban; Riva, rivaroxaban; Dabi, dabigatran; F, factor.

428 cases (98.4\%) as correct negative and in $7 / 428(1.6 \%)$ cases as false positive after comparison with the LC-MS/MS results ( - Table $\mathbf{3}$ ).

Thrombin inhibitor DOAC Dipstick test: In the DTI group, the thrombin inhibitor pad was evaluated in 427/429 cases (99.5\%) as correct positive and in $2 / 429$ cases $(0.5 \%)$ as false negative after comparison with the LC-MS/MS results. In the
DXI group, the thrombin inhibitor pad was evaluated in 448 / 451 cases (99.3\%) as correct negative and in 3/451 cases $(0.7 \%)$ as false positive after comparison with the LC-MS/MS results ( - Table 4 ).

The specificity, accuracy, and positive predictive value were significant at $95 \%(p<0.002)$ and the sensitivity and negative predictive value were significantly noninferior at a 
Table 1 Demographic data, concomitant diseases, and concomitant medication (multiple designations) of patients of DXI group and of DTI group

\begin{tabular}{|c|c|c|}
\hline & DXI group & DTI group \\
\hline \multicolumn{3}{|l|}{ Demographic data } \\
\hline$n$ & 451 & 429 \\
\hline Sex, male/female, $n / n$ & $250 / 201$ & $291 / 138$ \\
\hline Age $(y)$, mean (SD) & $67.3(14.1)$ & $70.6(11.6)$ \\
\hline Height $(\mathrm{cm})$, mean (SD) & $172.4(9.9)$ & $173.7(10.3)$ \\
\hline BMI $\left(\mathrm{kg} / \mathrm{m}^{2}\right)$, mean (SD) & $28.3(5.4)$ & $27.6(4.6)$ \\
\hline Apixaban, $n(\%)$ & $170(37.7)$ & \\
\hline Edoxaban, $n(\%)$ & $131(29.0)$ & \\
\hline Rivaroxaban, $n(\%)$ & $150(33.3)$ & \\
\hline Dabigatran, $n(\%)$ & & $429(100)$ \\
\hline Atrial fibrillation, $n(\%)$ & $287(63.6)$ & $391(91.1)$ \\
\hline VTE, $n(\%)$ & $136(30.2)$ & $17(4.0)$ \\
\hline Other indications, $n(\%)$ & $28(6.2)$ & $21(4.9)$ \\
\hline Secondary diagnoses & $n$ & $N$ \\
\hline Hypertension & 74 & 60 \\
\hline Diabetes & 20 & 13 \\
\hline Cardiac insufficiency & 14 & 20 \\
\hline Ischemic stroke & 18 & 27 \\
\hline Malignancy & 18 & 15 \\
\hline Thrombophilia & 32 & 3 \\
\hline Thrombotic disease & 44 & 13 \\
\hline Hemorrhagic disorder & 8 & 7 \\
\hline Rhythmologic intervention & 111 & 125 \\
\hline Cardiovascular disease & 104 & 141 \\
\hline Aortic stenosis & 32 & 58 \\
\hline Metabolic disease & 31 & 39 \\
\hline Neurologic disease & 5 & 36 \\
\hline Renal insufficiency & 19 & 7 \\
\hline Other disease & 85 & 83 \\
\hline Pulmonary disease & 7 & 15 \\
\hline \multicolumn{3}{|l|}{ Other medication } \\
\hline Platelet inhibitors & 72 & 66 \\
\hline Antidiabetic drugs & 19 & 23 \\
\hline Antihypertonic drugs & 239 & 205 \\
\hline Antiarrhythmic drugs & 12 & 47 \\
\hline Diuretic drugs & 43 & 56 \\
\hline Lipid lowering drugs & 37 & 55 \\
\hline Unspecific cardiac drugs & 13 & 23 \\
\hline Chemotherapy & 11 & 8 \\
\hline Hormones & 42 & 31 \\
\hline Gastrointestinal drugs & 33 & 32 \\
\hline Neurological drugs & 18 & 32 \\
\hline Other medications & 64 & 56 \\
\hline
\end{tabular}

Abbreviations: BMI, body mass index; DTI, direct thrombin inhibitor; DXI, direct factor Xa inhibitor; SD, standard deviation; VTE, venous thromboembolism.
Table 2 The concentrations of apixaban, edoxaban, rivaroxaban, and dabigatran in patient urine samples are shown as median values together with 5 th and 95 th percentiles

\begin{tabular}{|l|l|l|l|l|}
\hline & $\boldsymbol{n}$ & $\begin{array}{l}\text { Median } \\
(\mathbf{n g} / \mathbf{m L})\end{array}$ & $\begin{array}{l}\text { 5th percentile } \\
(\mathbf{n g} / \mathbf{m L})\end{array}$ & $\begin{array}{l}\text { 95th percentile } \\
(\mathbf{n g} / \mathbf{m L})\end{array}$ \\
\hline Apixaban & 170 & 648 & 89 & 3,213 \\
\hline Edoxaban & 131 & 8,785 & 417 & 71,203 \\
\hline Rivaroxaban & 150 & 1,903 & 248 & 8,160 \\
\hline Dabigatran & 429 & 4,206 & 515 & 21,642 \\
\hline
\end{tabular}

Table 3 Visual interpretation of the factor Xa inhibitor pad (pad 3) after incubation in urine samples from subjects in the direct oral factor Xa inhibitor (DXI) group and the direct oral thrombin inhibitor (DTI) group compared with LC-MS/MS results

\begin{tabular}{|l|l|l|l|}
\hline \multicolumn{3}{|l|}{ Factor Xa inhibitor pad } & DOAC Dipstick result \\
\hline \multicolumn{2}{|l|}{ LC-MS/MS result } & Positive & Negative \\
\hline \multicolumn{2}{|l|}{} & 435 & 17 \\
\hline Positive & 452 & 7 & 421 \\
\hline Negative & 428 & 442 & 438 \\
\hline Sum & 880 & & \\
\hline
\end{tabular}

Abbreviations: DOAC, direct oral anticoagulant; LC-MS/MS, liquid chromatography-tandem mass spectrometry.

Table 4 Visual evaluation of thrombin inhibitor pad (pad 4) after incubation in urine samples from subjects in the direct oral factor Xa inhibitor (DXI) group and the direct oral thrombin inhibitor (DTI) group compared with LC-MS/MS results

\begin{tabular}{|l|l|l|l|}
\hline \multicolumn{3}{|l|}{ Thrombin inhibitor pad } & \multicolumn{2}{l|}{ DOAC Dipstick result } \\
\hline \multicolumn{2}{|l|}{ LC-MS/MS result } & Positive & Negative \\
\hline \multicolumn{2}{|l|}{} & 427 & 2 \\
\hline Positive & 429 & 3 & 448 \\
\hline Negative & 451 & 430 & 450 \\
\hline Sum & 880 & 430 \\
\hline
\end{tabular}

Abbreviations: DOAC, direct oral anticoagulant; LC-MS/MS, liquid chromatography-tandem mass spectrometry.

proportion of $95 \%$ including the $0.5 \%$ margin $(p<0.04)$. The agreement (kappa value) between results of the factor Xa inhibitor pad and LC-MS/MS was 0.945 (-Table 5).

The results for sensitivity, specificity, accuracy, and negative and positive predictive visual evaluation of the thrombin inhibitor pad were significant at a proportion of $97.5 \%$ $(p<0.001)$. The kappa value was 0.987 (-Table 5).

The individual concentrations of the DOACs determined by LS-MS/MS are reported in -Supplementary Tables $\mathbf{S 1}$ (DXI group) and $\mathbf{5 2}$ (DTI group) (available in the online version) for subjects in whom observers assessed colors of pad 3 (factor Xa inhibitors) and pad 4 (thrombin inhibitor) as false negative or false positive. The urine color pad was observed as "normal" in these cases. Therefore, the observers had probably interpreted the colors of the dipstick incorrectly. 
Table 5 Sensitivity, specificity, accuracy, negative predictive values (NPV), positive predictive values (PPV), and kappa index of the visual evaluation of factor Xa inhibitor pad and of thrombin inhibitor pad (mean, 95\% confidence interval)

\begin{tabular}{|l|l|l|l|l|}
\hline & \multicolumn{2}{|l|}{$\begin{array}{l}\text { Factor Xa inhibitor } \\
\text { pad }\end{array}$} & \multicolumn{2}{l|}{$\begin{array}{l}\text { Thrombin inhibitor } \\
\text { pad }\end{array}$} \\
\hline & Mean & $95 \% \mathrm{Cl}$ & Mean & $95 \% \mathrm{Cl}$ \\
\hline Sensitivity & 0.962 & $0.941 ; 0.978$ & 0.995 & $0.983 ; 0.999$ \\
\hline Specificity & 0.984 & $0.967 ; 0.993$ & 0.991 & $0.978 ; 0.998$ \\
\hline Accuracy & 0.973 & $0.960 ; 0.982$ & 0.993 & $0.985 ; 0.998$ \\
\hline NPV & 0.961 & $0.939 ; 0.977$ & 0.996 & $0.984 ; 0.999$ \\
\hline PPV & 0.984 & $0.968 ; 0.994$ & 0.991 & $0.976 ; 0.998$ \\
\hline Kappa & 0.945 & $0.924 ; 0.967$ & 0.987 & $0.976 ; 0.997$ \\
\hline
\end{tabular}

Abbreviations: $\mathrm{Cl}$, confidence interval; na, not available.

Receiver operating curves demonstrated $c$-values of 0.989 for the factor Xa inhibitor pad ( $\mathbf{- F i g . ~ 2 A )}$ ) and 0.995 for the thrombin inhibitor pad (-Fig. 2B). (A $c$-value of 0.5 indicates that a test result is not better than chance, and a $c$-value of 1.0 indicates a "perfect" test result.)

\section{Intention-to-Analyze Evaluation}

The intention-to-analyze evaluation confirmed the results of the per-protocol evaluation ( $\mathbf{- T a b l e s} 6-\mathbf{8}$ ). There were no differences of these results with those of the per-protocol evaluation.

\section{Center Effects}

Relevant differences in visual evaluation of the factor Xa inhibitor and thrombin inhibitor pads were not detected
Table 6 Intention-to-analyze evaluation: visual evaluation of the factor Xa inhibitor pad (pad 3) after incubation in urine samples from subjects in the oral direct factor Xa inhibitor (DXI) group and the oral thrombin inhibitor (DTI) group compared with LC-MS/MS results

\begin{tabular}{|l|l|l|l|}
\hline \multicolumn{3}{|l|}{ Factor Xa inhibitor pad } \\
\hline \multicolumn{2}{|l|}{ LC-MS/MS result } & \multicolumn{3}{l|}{ DOAC Dipstick result } \\
\hline \multicolumn{2}{|l|}{} & Positive & Negative \\
\hline Positive & 460 & 442 & 18 \\
\hline Negative & 445 & 11 & 434 \\
\hline Sum & 905 & 453 & 452 \\
\hline
\end{tabular}

Abbreviations: DOAC, direct oral anticoagulant; LC-MS/MS, liquid chromatography-tandem mass spectrometry.

between centers. For the factor Xa inhibitor pad, 24/880 $(2.8 \%)$ colors were interpreted falsely (-Supplementary Table S3 [available in the online version], $p=0.518$; chisquare test). The thrombin inhibitor pad colors were interpreted falsely in 5 of 880 cases (0.7\%) (-Supplementary Table 54, available in the online version). Differences between centers were not calculated because of the low frequencies ( $n=0$ or $n=1$ ) per center.

\section{Other Findings}

The urine color pad was evaluated as abnormal in three cases and, consequently, the results were excluded from the evaluation (see - Fig. 1). Seven of 451 results in the DXI group and 3 of 429 results in the DTI group were DOAC concentrations $<30 \mathrm{ng} / \mathrm{mL}$ suggesting that participants had not taken their

\section{Factor Xa Inhibitor Pad}

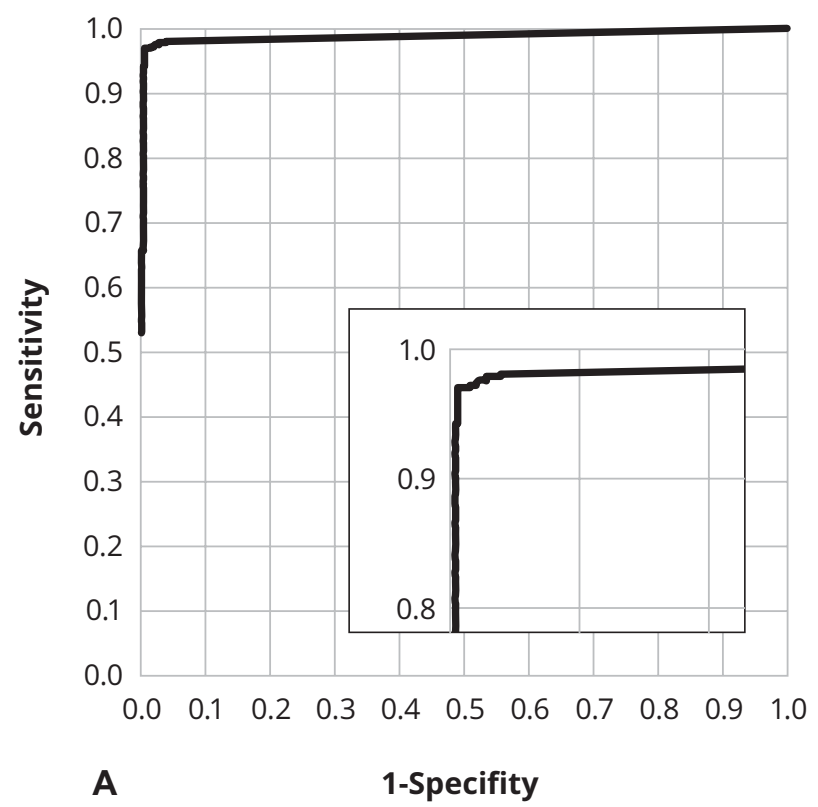

Thrombin Inhibitor Pad

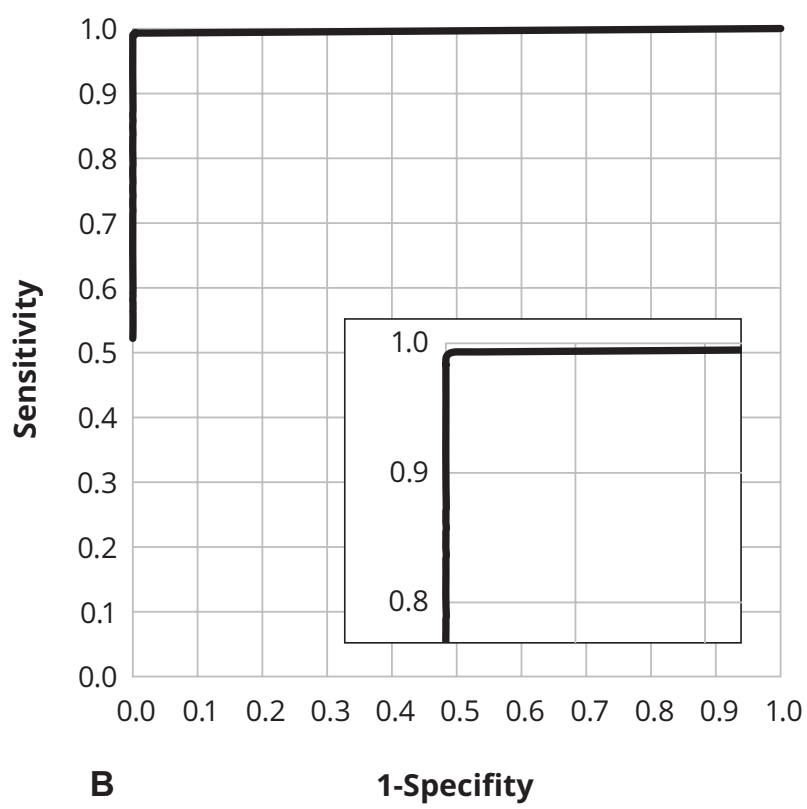

Fig. 2 Receiver-operating curves (ROCs) of visual evaluation of the factor Xa inhibitor pad (A) and the thrombin inhibitor pad (B). Inlet: magnification of the "critical" part of the ROC. 
Table 7 Intention-to-analyze evaluation: visual evaluation of the thrombin inhibitor pad (pad 4) after incubation in urine samples from subjects in the direct oral factor Xa inhibitor (DXI) group and the direct oral thrombin inhibitor (DTI) group compared with LC-MS/MS results

\begin{tabular}{|l|l|l|l|}
\hline \multicolumn{3}{|l|}{ Thrombin inhibitor pad } \\
\hline \multicolumn{2}{|l|}{ LC-MS/MS result } & DOAC Dipstick result \\
\hline \multicolumn{2}{|l|}{} & Positive & Negative \\
\hline Positive & 438 & 436 & 2 \\
\hline Negative & 468 & 5 & 463 \\
\hline Sum & 906 & 441 & 465 \\
\hline
\end{tabular}

Table 8 Intention-per-protocol analysis: sensitivity, specificity, accuracy, negative predictive value (NPV), and positive predictive value (PPV) of visual evaluation of the factor Xa inhibitor pad and the thrombin inhibitor pad

\begin{tabular}{|l|l|l|l|l|}
\hline & \multicolumn{2}{|l|}{$\begin{array}{l}\text { Factor Xa inhibitor } \\
\text { pad }\end{array}$} & \multicolumn{2}{l|}{$\begin{array}{l}\text { Thrombin inhibitor } \\
\text { pad }\end{array}$} \\
\hline & Mean & $95 \% \mathrm{Cl}$ & Mean & $95 \% \mathrm{Cl}$ \\
\hline Sensitivity & 0.961 & $0.939 ; 0.977$ & 0.993 & $0.980 ; 0.999$ \\
\hline Specificity & 0.975 & $0.956 ; 0.988$ & 0.991 & $0.983 ; 0.999$ \\
\hline Accuracy & 0.968 & $0.957 ; 0.978$ & 0.992 & $0.984 ; 0.997$ \\
\hline NPV & 0.958 & $0.953 ; 0.975$ & 0.994 & $0.981 ; 0.999$ \\
\hline PPV & 0.976 & $0.957 ; 0.988$ & 0.991 & $0.977 ; 0.998$ \\
\hline Kappa & 0.936 & $0.913 ; 0.959$ & 0.985 & $0.973 ; 0.996$ \\
\hline
\end{tabular}

Abbreviation: $\mathrm{Cl}$, confidence interval.

medication; these results were nonetheless included in the intention-to-analyze evaluation.

\section{Discussion}

The results of this study describe for the first time a multicenter evaluation of a rapid point-of-care test to determine if DOACs are present in a patient's urine sample. The accuracy of tests for Xa and thrombin inhibitors were 97.3 and $99.3 \%$ and sensitivities, specificities, and negative and positive predictive values were all $95 \%$ or higher with a tendency of higher accuracy values obtained with the thrombin inhibitor DOAC Dipstick pad. Previously reported sensitivity and specificity values of point-of-care devices using prothrombin time, activated partial thromboplastin time, or diluted clotting time reagents and whole blood samples ranged between 60 and $95 \%$ for each of the two types of DOACs. ${ }^{18-20}$ The multicenter study collected a large sample and the intercenter variation in interpretations was low, demonstrating the feasibility of the assay.

Strengths of this study and its results can be summarized as follows: The test results demonstrate at least 95\% correct positive and correct negative results for both oral factor Xa and thrombin inhibitor types of DOACs. The test is easy and rapid to perform and the results can be interpreted by comparison with a predefined color scale. Sample collection for the test is usually not invasive, although urine catheterization may be needed to obtain a sample from unconscious patients. The test can be repeated if the result is unclear and can be performed at any time without technical equipment. If patients are unable to communicate the type of anticoagulants/DOAC they have taken, the DOAC Dipstick may indicate whether specific DOAC testing, such as antifactor Xa or antithrombin laboratory tests, should be ordered. Because antithrombin is absent in normal human urine, the test does not interfere with heparins ${ }^{7}$ unlike most assays using blood samples. ${ }^{18}$

How can the test be implemented into the clinical setting of patient care based on the results presented here? Importantly, the test result can be interpreted only in connection with the patient's clinical situation. Examples of patients in whom testing may be beneficial are for example, if a patient presents with a major hemorrhage and the DOAC Dipstick test result is negative, DOACs are highly unlikely to contribute to the bleeding event. This allows medical treatment to be administered immediately without waiting for results of quantitative DOAC tests. If the DOAC Dipstick test gives a positive result in a patient presenting with major haemorrhage, then a specific antidote can be considered immediately or after plasma DOAC levels have been determined. In another scenario, if a patient on a DOAC therapy requires an urgent major operative procedure and the DOAC Dipstick test is negative, it is unlikely that the patient has significant DOAC concentrations in blood, and the operative procedure may be performed as soon as indicated. However, if the DOAC Dipstick test is positive, the operation may need to be postponed or additional blood tests have been completed to guide the decision process. The test may be used before epidural anesthesia or other interventions with high bleeding risk to identify patients with residual DOAC activity, which would put the patient at high risk of hemorrhages. In patients with ischemic stroke, a negative test result for both types of DOACs may be relevant for consideration to give thrombolytic therapy. Furthermore, the DOAC Dipstick test may confirm if a patient has stopped intaking their DOACs, which may indicate a required temporary interruption or noncompliance.

The present study has some limitations. The time since last emptying of the urine bladder may influence the amount of DOAC present in urine, but this issue was not specifically considered or standardized in the present study. Although false negative results are rare, they may occur when a patient has taken his first DOAC immediately before urine sampling. Studies are ongoing to determine a correlation between blood and urine DOAC concentration. ${ }^{21,22}$ Purple urine ${ }^{23}$ and other abnormal urine colors ${ }^{24}$ may influence the visual assessment of the test strip. To address this, a "urine color" test pad that does not contain reagents was included in the dipstick device to detect abnormal urine color and avoid misinterpretation of the DOAC test pads. ${ }^{23}$ The excretion of DOACs into urine is reduced with impairment of renal function. Creatinine is also excreted less into urine when renal function is impaired, so a fourth test pad was added to the Dipstick device. If this pad indicates little or no creatinine in the urine sample, then the DOAC test result should be considered invalid. 
In summary, the present study shows that DOAC Dipstick test sensitively and specifically determines the presence of both Xa and IIa inhibitors in urine samples, if compared with the gold standard of LC-MS/MS. The evaluation of the DOAC Dipstick test in emergency medicine and other patient groups is ongoing.

\section{What is known about this topic?}

- In specific patient groups rapid determination of the presence or absence of DOAC is required to facilitate the medical decision-making processes.

- Currently available global and point-of-care coagulation assays have limitations. Specific assays are only possible in specialized laboratories and cannot be completed quickly enough in urgent situations.

- DOACs are excreted into urine and their presence or absence can be determined within 10 minutes by a test strip.

\section{What does this paper add?}

- This multicenter study showed that factor Xa inhibitors (apixaban, edoxaban, rivaroxaban) and the thrombin inhibitor dabigatran were detected with high sensitivity, specificity, and accuracy in patient urine samples using a rapid DOAC Dipstick point-of-care test.

- The high negative and positive predictive values of the test can be integrated into laboratory and clinical decision-making processes and may improve patient care.

Participating Centers (alphabetical order per town, number of included patients in brackets)

Bad Krozingen, Universitätsherzzentrum, Klinik für Kardiologie und Angiologie II, Prof. Dr. Dietmar Trenk, Prof. Dr. Julia Hromek, Dr. Stefan Leggewie $(n=232)$; Bad Oeynhausen, Herz - und Diabeteszentrum NRW, Klinik für Kardiologie, Prof. Dr. Cornelia Piper, Dr. Isgandarova Potratz $(n=79)$; Berlin, Vivantes Klinikum Neukölln, Kardiologie, Prof. Dr. Harald Darius, Dr. Lorenz Reill $(n=36)$; Berlin, Vivantes Klinikum im Friedrichshain, Zentrum für Gefäßmedizin - Sprechstunde für Gerinnungsstörung und Hämophiliezentrum, Priv. Doz Dr. Robert Klamroth, Dr. Cornelia Kubicek-Hofmann, Dr. Ana Marija Orlovic ( $n=27)$; Berlin, Gerinnungszentrum Berlin, Priv.-Doz. Dr . Christoph Sucker $(n=46)$; Coburg, II. Medizinische Klinik - Innere Medizin - Kardiologie, Dr. Steffen Schnupp, Dr. Issameddine Ajimi, Dr. Christian Mahnkopf, Dr. Thomas Mischke $(n=70)$; Darmstadt, Klinik für Gefäßmedizin - Angiologie, Prof. Dr Rupert Bauersachs, Priv. Doz. Dr. Jörg Herold $(n=18)$; Dessau-Roßlau, Praxis für Innere Medizin, Kardiologie, Angiologie, Dr. Frank Menzel, Frank $(n=41)$; Dresden, Studienambulanz Klinische Thromboseforschung, Priv. Doz. Dr. Jan Beyer-Westendorf, Dr. Sandra Marten,
Dr. Christiane Naue $(\boldsymbol{n}=3)$; Dresden, Studienambulanz der GWT - TUD GmbH, Prof. Dr. Sebastian Schellong, Dr. Carsten Müller $(n=12)$; Frankfurt, CCB Studienzentrum $\mathrm{GmbH}$, Prof. Dr. Edelgard Lindhoff-Last, Dr. Barbara Zydek $(n=89)$; Freiburg, Klinik für Kardiologie und Angiologie I, Prof. Dr Daniel Dürschmied, Dr. Roland Giesen, Dr. Christoph Olivier $(n=12)$; Hamburg, Onkologisches Zentrum, II. Medizinische Klinik und Poliklinik, Gerinnungsambulanz, Prof. Dr. Florian Langer, Dr. Minna Voigtländer, $(n=16)$; Heidenheim, Facharztpraxis für Innere Medizin, Schwerpunkt Hämatologie, Onkologie und Hämostaseologie, Priv. Doz. Dr. Martin Grünewald $(n=14)$; Köln, Klinik für Kardiologie und internistische Intensivmedizin, Prof. Dr. Ingo Ahrens, Ingo, Dr. Timo Bömicke $(n=2)$; Leipzig, Zentrum für Blutgerinnungsstörungen, Dr. Ute Scholz, Dr. Michael Krause $(n=18)$; Stahnsdorf, Parkkardiologie, Dr. Ulrich Wolf $(n=129)$; Siegen, Zentrum für Prävention und Rehabilitation, Dr. Ulrich Overhoff, Dr. Fabian Krämer $(n=68)$.

\section{Conflict of Interest}

J.H. is managing director and founder of DOASENSE and reports personal fees from Portola, outside the submitted work; a patent US 9,133,501 licensed, a patent EU 2643475 licensed, a patent US 9,944,971 licensed, and a patent EU 2723886 licensed. J.B.W. reports grants and personal fees from Bayer, grants and personal fees from Daiichi Sankyo, grants and personal fees from DOASENSE, grants and personal fees from Portola, grants from Pfizer, during the conduct of the study. P.V. reports grants and personal fees from Bayer, Boehringer Ingelheim, BMS, Pfizer, Daiichi-Sankyo and Portola, outside the submitted work. J.D. is the CEO and founder of QUALIblood and reports personal fees from Diagnostica Stago, Roche, Roche Diagnostics, Portola, and Daiichi-Sankyo, outside the submitted work. C.W. reports research grant and consultancy from DOASENSE during the conduct of the study. S.H. reports research grant from DOASENSE during the conduct of the study. M.C. reports grants and other from Bayer, personal fees from Shionogi, Alexion, Pfizer, Octapharma, BMS Canada, CSL Behring, Servier Canada, Diagnostica Stago, and Asahi Kasei, outside the submitted work; grants from Leo Pharma and Heart and Stroke Foundation, and other from Daiichi and Alnylam. I.E. reports grants and personal fees from Stago, Boehringer-Ingelheim, Bayer, Pfizer, Bristol Myers Squibb, during the conduct of the study; grants and personal fees from Stago, Boehringer Ingelheim, Pfizer, Bristol Myers Squibb, Bayer, outside the submitted work. R.B. reports personal fees from DOASENSE GmbH, during the conduct of the study; personal fees from Bayer AG, BMS, Daichii-Sankyo, Pfizer, outside the submitted work.

\section{Acknowledgments}

The authors are grateful to the study participants listed above. We also wish to thank Simone Mangold and Miriam Weiss for their help conducting the study, and Rupert Schreiner for performing the LC-MS/MS analyses. 


\section{References}

1 Steffel J, Verhamme P, Potpara TS, et al; ESC Scientific Document Group. The 2018 European Heart Rhythm Association Practical Guide on the use of non-vitamin K antagonist oral anticoagulants in patients with atrial fibrillation. Eur Heart J 2018;39(16):1330-1393

2 Schünemann HJ, Cushman M, Burnett AE, et al. American Society of Hematology 2018 guidelines for management of venous thromboembolism: prophylaxis for hospitalized and nonhospitalized medical patients. Blood Adv 2018;2(22):3198-3225

3 Spyropoulos AC, Al-Badri A, Sherwood MW, Douketis JD. To measure or not to measure direct oral anticoagulants before surgery or invasive procedures: comment. JThromb Haemost 2016;14(12):2556-2559

4 Tripodi A. To measure or not to measure direct oral anticoagulants before surgery or invasive procedures. JThromb Haemost 2016; 14(07):1325-1327

5 Ebner M, Birschmann I, Peter A, et al. Limitations of specific coagulation tests for direct oral anticoagulants: a critical analysis. J Am Heart Assoc 2018;7(19):e009807

6 Bluecher A, Meyer Dos Santos S, Ferreirós N, et al. Microfluidic coagulation assay for monitoring anticoagulant therapy in acute stroke patients. Thromb Haemost 2017;117(03):519-528

7 Harenberg J, Schreiner R, Hetjens S, Weiss C. Detecting anti-IIa and anti-Xa direct oral anticoagulant (DOAC) agents in urine using a DOAC Dipstick. Semin Thromb Hemost 2019;45(03):275-284

8 Kim PY, Di Giuseppantonio LR, Wu C, Douketis JD, Gross PL. An assay to measure levels of factor Xa inhibitors in blood and plasma. J Thromb Haemost 2019;17(07):1153-1159

9 Ten Cate H, Henskens YM, Lancé MD. Practical guidance on the use of laboratory testing in the management of bleeding in patients receiving direct oral anticoagulants. Vasc Health Risk Manag 2017;13:457-467

10 Douxfils J, Gosselin RC. Laboratory assessment of direct oral anticoagulants. Semin Thromb Hemost 2017;43(03):277-290

11 Harenberg J, Krämer S, Du S, Weiss C, Krämer R. Concept of a point of care test to detect new oral anticoagulants in urine samples. Thromb J 2013;11(01):15

12 Harenberg J, Du S, Krämer S, Weiss C, Krämer R, Wehling M. Patients' serum and urine as easily accessible samples for the measurement of non-vitamin $\mathrm{K}$ antagonist oral anticoagulants. Semin Thromb Hemost 2015;41(02):228-236

13 Schreiner R, Hetjens S, Giese C, et al. Determination of rivaroxaban, apixaban, edoxaban and dabigatran by liquid chromatogra- phy-tandem massspectrometry and chromogenic assays from urine samples of patients. Res Pract Thromb Haemost 2017;1:491

14 Levy JH, Ageno W, Chan NC, Crowther M, Verhamme P, Weitz JI; Subcommittee on Control of Anticoagulation. When and how to use antidotes for the reversal of direct oral anticoagulants: guidance from the SSC of the ISTH. JThromb Haemost 2016;14 (03):623-627

15 Albaladejo P, Pernod G, Godier A, et al; members of the French Working Group on Perioperative Haemostasis. Management of bleeding and emergency invasive procedures in patients on dabigatran: updated guidelines from the French Working Group on Perioperative Haemostasis (GIHP) - September 2016. Anaesth Crit Care Pain Med 2018;37(04):391-399

16 Wiegele M, Schöchl H, Haushofer A, et al. Diagnostic and therapeutic approach in adult patients with traumatic brain injury receiving oral anticoagulant therapy: an Austrian interdisciplinary consensus statement. Crit Care 2019;23(01):62

17 Landis JR, Miller ME, Davis CS, Koch GG. Some general methods for the analysis of categorical data in longitudinal studies. Stat Med 1988;7(1-2):109-137

18 Ebner M, Birschmann I, Peter A, et al. Limitations of specific coagulation tests for direct oral anticoagulants: a critical analysis. J Am Heart Assoc 2018;7(19):e009807

19 Gosselin RC, Adcock DM, Bates SM, et al. International Council for Standardization in Haematology (ICSH) recommendations for laboratory measurement of direct oral anticoagulants. Thromb Haemost 2018;118(03):437-450

20 Cuker A, Burnett A, Triller D, et al. Reversal of direct oral anticoagulants: Guidance from the Anticoagulation Forum. Am J Hematol 2019;94(06):697-709

21 Merrelaar A, Buchtele N, Schörgenhofer C, et al. Dark-colored urine affects test results of the novel DOAC Dipstick point-of-care strip test. Res Pract Thromb Haemost 2019:PB 181

22 Tafur A, Harenberg J, Walenga J, et al. Accuracy of DOASENSE Dipstick for assessing patients treated peri-operatively with DOACs - pilot study. Res Pract Thromb Haemost 2019:PB 179

23 Gulmez MD, Yildirim O, Soyhan M, et al. Purple urine: an astonishing symptom in an elderly patient with acute kidney injury. Intern Med J 2019;49(05):677-679

24 Dicko A, Roh ME, Diawara H, et al. Efficacy and safety of primaquine and methylene blue for prevention of Plasmodium falciparum transmission in Mali: a phase 2, single-blind, randomised controlled trial. Lancet Infect Dis 2018;18(06):627-639 